Lynch P.B., 1989. Voluntary food intake of sows and gilts. In : A.T. Smith and T.L.J. Lawrence (eds), Pig housing and the environment, 71-77. Brit. Soc. Anim. Prod., Edinburgh.

McGlone J.J., Stansbury W.F., Tribble L.F., 1988. Management of the lactating sow during heat stress : effects of water dript, snout coolers, floor type and a high energy-density diet. J. Anim. Sci., 66, 885-891.

Messias de Bragança M., Quesnel H., Mounier A.-M., Prunier A., 1995. Influence de la température ambiante sur les performances zootechniques et certains paramètres sanguins chez des truies Large White primipares. Journées Rech. Porcine en France, 27, 37-44.

Moser R.L., Cornelius S.G., Pettigrew J.E., Hanke H.E., Heeg T.R., Miller K.P., 1987. Influence of postpartum feeding method on performance of the lactating sow. Livest. Prod. Sci., 16, 91-99.

Neil M., 1996. Ad libitum lactation feeding of sows introduced immediately before, at, or after farrowing. Anim. Sci., 63, 497-505.

Noblet J., Le Dividich J., Bikawa T., 1985. Interaction between energy level in the diet and environmental temperature on the utilization of energy in growing pigs. J. Anim. Sci., 61, 452-459.
O'Grady J.F., Lynch P.B., 1978. Voluntary feed intake by lactating sows : influence of system of feeding an nutrient density of the diet. Ir. J. Agric. Res., $17,1-5$

O’Grady J.F., Lynch P.B., Kearney P.A., 1985. Voluntary feed intake by lactating sows. Livest. Prod. Sci. $12,355-365$.

Prunier A., Dourmad J.-Y., Etienne M., 1994. Effect of light regimen under various ambiant temperatures on sow and litter performance. J. Anim. Sci., $72,1461-1466$

Schœnherr W.D., Stahy T.S., Cromwell G.L., 1989 The effects of dietary fat or fiber addition on yield and composition of milk from sows housed in warm or hot environment. J. Anim. Sci., 67, 482-495.

Trottier N.L., Easter R.A., 1995. Dietary and plasma branched-chain amino acids in relation to tryptophan : effect on voluntary feed intake and lactation metabolism in the primiparous sow. J. Anim. Sci. 73, 1086-1092.

Verstegen M.W.A., Mesu J., van Kempen G.J.M., Geerse C., 1985. Energy balances of lactating sows in relation to feeding level and stage of lactation. $\mathrm{J}$. Anim. Sci., 60, 731-740.

\section{ETIENNE, \\ M.-C. PERE}

INRA Station

de Recherches

Porcines, 35590 Saint-Gilles

\title{
Adaptations physiologiques et métaboliques au cours de la gestation chez la truie
}

\section{Apports nutritionnels et performances de reproduction}

Les quantités d'énergie ou de protéines allouées aux truies gravides n'affectent pas en général la taille de la portée. En revanche, l'augmentation des apports énergétiques accroît le poids du porcelet à la naissance (Henry et Etienne 1978). L'effet est plus marqué chez les truies multipares $(+37 \mathrm{~g} / \mathrm{Mcal} ; \mathrm{R}$ $=0,65)$ que chez les primipares $(+15 \mathrm{~g} /$ Mcal ; $\mathrm{R}=0,30)$. Seules des conditions extrêmes d'apports de protéines (aliments composés quasi exclusivement de céréales régimes protéiprives) provoquent une réduction de poids des porcelets nouveau-nés (Duée et Sève 1978).

\section{Adaptations maternelles et fœtales}

\section{Capacité utérine}

La truie n'est capable de conduire à terme qu'un nombre limité de fotus. Cette limitation, appelée capacité utérine, est caractéris- tique du type génétique et de l'individu. Différents modèles permettant de faire varier le nombre potentiel d'embryons ont été utilisés. La régularisation de la taille de la portée intervient précocement, généralement avant 35 jours de gestation. Cependant, lorsque l'effectif potentiel (taux d'ovulation) est important, des différences persistent à ce stade, et la mortalité tardive des fotus est accrue. Les effets d'une augmentation ou d'une restriction de l'espace utérin disponible par fotus sur le développement de la portée ont été étudiés en comparant des truies témoins (CTR), des femelles HHO ayant subi une hémi-hystéroovariectomie droite (l'effectif initial d'embryons dans la corne utérine subsistante est deux fois plus important que dans chacune des cornes des truies du lot CTR), et des truies LIG dont l'oviducte gauche était ligaturé (l'effectif initial d'embryons dans chacune des deux cornes utérines est deux fois plus faible que celui du lot CTR). La figure 1 présente l'évolution du nombre de fœtus par corne utérine pendant la gestation dans les trois groupes expérimentaux. A 35 jours de gestation, la hiérarchie du nombre de fotus par corne utérine est la même qu'au début, mais les écarts entre les groupes sont fortement atténués en raison d'une mortalité 
Figure 1. Evolution au cours de la gestation du nombre de foetus par corne utérine (Père et al 1995). LIG, CTR et HHO : voir texte.

$\begin{array}{ll}\square & \text { Taux d'ovulation } \\ \square & \text { Foetus à } 35 \text { jours } \\ \square & \text { Foetus à } 112 \text { jours }\end{array}$

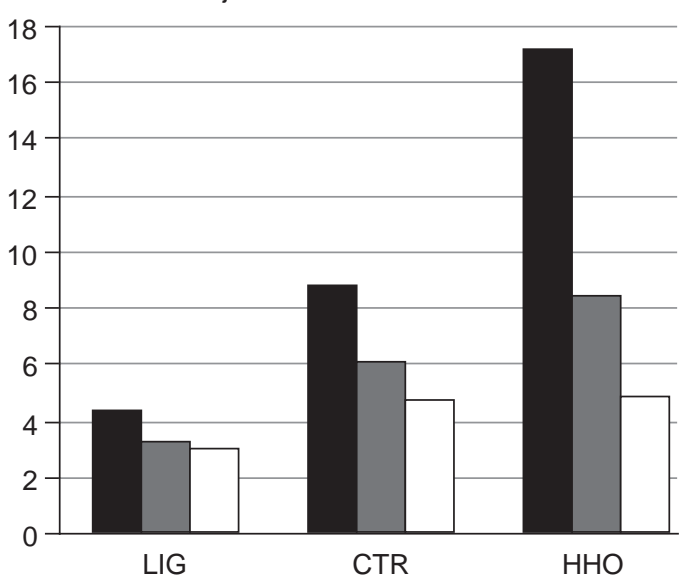

embryonnaire précoce d'autant plus élevée que le nombre initial d'embryons était important. Cet effet se poursuit par la suite, l'écart entre les groupes CTR et HHO ayant disparu en fin de gestation. Le poids moyen des porcelets à la naissance dépend de leur effectif en début de gestation, ce qui indique que la compétition entre les fotus intervient tout au long de la gestation. Dans l'exemple précédent, il était respectivement de 1,50, 1,38 et $1,27 \mathrm{~kg}$ dans les lots LIG, CTR et HHO. On n'a donc pas intérêt à accroître le taux d'ovulation au-delà de celui qui est nécessaire pour maximiser la taille de la portée.

\section{Apports nutritionnels aux fœetus}

Les apports nutritionnels aux fœetus dépendent notamment du débit sanguin et des concentrations des substrats au niveau de l'utérus gravide. Ces facteurs ont été étudiés en recourant aux modèles utilisés pour mettre en évidence la capacité utérine, qui permettent de faire varier la taille de la portée et la croissance des fotus.

Chez la truie, le débit sanguin utérin augmente de façon linéaire au cours de la gestation (Père et al 1996). Il est d'autant plus élevé que l'effectif de fœtus dans la corne utérine est plus important : il passe de 0,8 à $1,7 \mathrm{l} / \mathrm{min} /$ corne utérine entre 44 et 111 jours de gestation chez les truies LIG, et de 1,2 à $2,8 \mathrm{l} / \mathrm{min}$ chez les truies CTR ou de 1,1 à $2,6 \mathrm{l} / \mathrm{min}$ chez les truies HHO. L'augmentation du débit sanguin utérin jusqu'au terme n'avait pas été montrée antérieurement chez la truie, vraisemblablement pour des raisons d'ordre méthodologique (Ford et al 1984, Reynolds et al 1985); elle est conforme à ce qui est trouvé dans les autres espèces. A tous les stades de mesure, le débit sanguin/fœtus est d'autant plus élevé que le nombre de fœtus est plus faible (figure 2). Ceci explique que les porcelets à la naissance soient d'autant moins lourds que la taille de portée est plus impor-
Figure 2. Effet du nombre de fœtus dans la corne utérine sur l'évolution du débit sanguin utérin par fœtus au cours de la gestation (Père et al 1996).

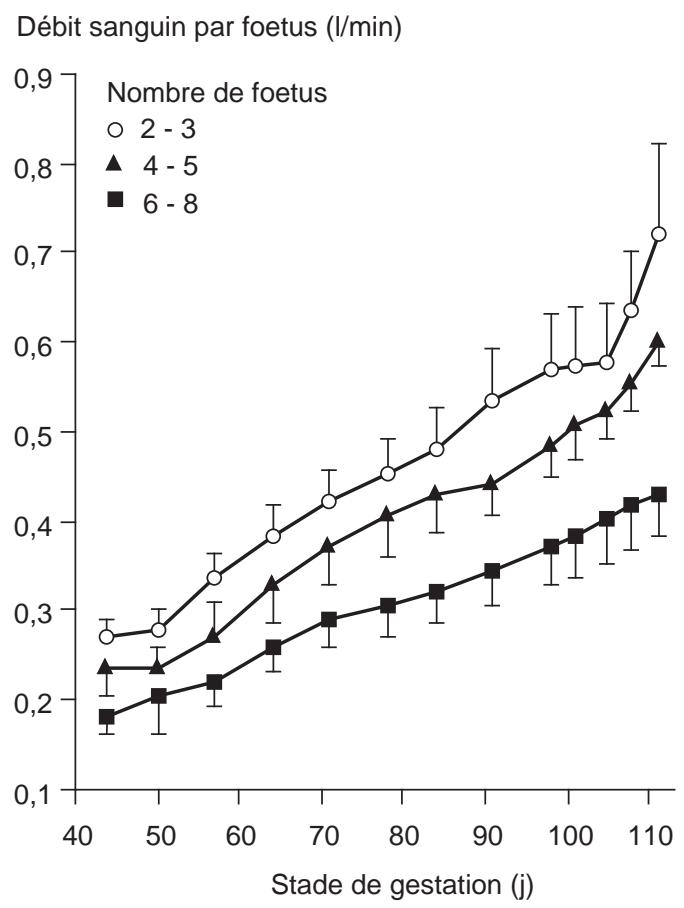

tante. L'augmentation du débit sanguin utérin pendant la gestation constitue donc une adaptation majeure aux besoins croissants de l'utérus gravide. Cette adaptation est cependant partielle, puisque le rapport débit sanguin/poids des fotus dans la corne passe de 1,77 à $0,39 \mathrm{ml} / \mathrm{min}$ par gramme entre 44 et 111 jours de gestation.

La disponibilité en substrats dans le sang maternel varie avec le stade de gestation et la taille de la portée in utero (tableau 1). Les concentrations de glucose, d'acides gras libres, et des substrats gluconéogéniques (glycérol, lactate, alanine), plus élevées à la fin qu'au milieu de la gestation, révèlent leur plus grande disponibilité pour l'utérus gravide. Ceci permet de répondre à l'accroissement des besoins du conceptus pendant la dernière partie de la gestation. Les concentrations de glucose sont plus faibles, et celles des acides gras libres et du glycérol plus élevées chez les

Tableau 1. Concentrations plasmatiques des substrats et de l'insuline (Père et al 1997).

\begin{tabular}{|l|c|c|c|c|c|c|}
\hline \multicolumn{1}{|c|}{ Lot } & \multicolumn{2}{c|}{ LIG } & \multicolumn{2}{c|}{ TEM } & \multicolumn{2}{c|}{ HHO } \\
\hline Stade de gestation, jours & 60 & 110 & 60 & 110 & 60 & 110 \\
\hline Taille de la portée $(112 \mathrm{j})$ & \multicolumn{2}{|c|}{5,8} & \multicolumn{2}{c|}{9,4} & \multicolumn{2}{c|}{4,8} \\
Poids de la portée $(\mathrm{kg})$ & \multicolumn{2}{|c|}{8,9} & \multicolumn{2}{c|}{12,9} & \multicolumn{2}{c|}{5,9} \\
Glucose $(\mathrm{mmol} / \mathrm{l})$ & 5,06 & 5,08 & 4,69 & 4,92 & 5,12 & 5,34 \\
Insuline $(\mu \mathrm{UI} / \mathrm{ml})$ & 26 & 7 & 11 & 5 & 18 & 11 \\
NEFA $(\mu \mathrm{mol} / \mathrm{l})$ & 109 & 262 & 174 & 447 & 118 & 291 \\
Glycérol $(\mu \mathrm{mol} / \mathrm{l})$ & 20 & 37 & 30 & 61 & 30 & 49 \\
Lactate $(\mathrm{mmol} / \mathrm{l})$ & 7,98 & 7,94 & 8,69 & 10,74 & 8,00 & 12,60 \\
Alanine $(\mu \mathrm{mol} / \mathrm{l})$ & 421 & 464 & 355 & 525 & 436 & 496 \\
Urée $(\mathrm{mmol} / \mathrm{l})$ & 2,88 & 3,16 & 2,80 & 3,30 & 2,82 & 3,01 \\
\hline
\end{tabular}


truies dont les portées sont de plus grande taille et de poids plus élevé. Le glucose semble ainsi davantage prélevé par l'utérus, et une lipolyse accrue se produit pour répondre aux besoins énergétiques de la mère. Tout ceci montre donc que des modifications métaboliques se mettent en place chez la truie pour s'adapter aux besoins de l'utérus gravide.

La placentation épithélio-choriale de la truie limite les échanges de substrats entre la mère et ses fotus. Comme dans les autres espèces étudiées, le glucose est le principal substrat énergétique pour le fotus de porc (Père 1995, tableau 2). Le transfert maternofotal du glucose est particulièrement faible dans cette espèce puisque la pente de la régression entre les glycémies fœtale et maternelle n'est que de 0,30 (Père 1997). Le lactate et le fructose, produits par le placenta, sont en concentration élevée chez le fotus ( 2,6 fois plus de lactate que chez la truie, et absence de fructose chez la mère). Les coefficients d'extraction sont respectivement de 14 et de $8 \%$ pour le glucose et le lactate au niveau ombilical. Contrairement à d'autres espèces (homme, cobaye), le passage transplacentaire des acides gras est extrêmement faible chez le porc.

\section{Sensibilité à l'insuline chez la truie}

Pour faire face à la demande croissante en glucose de la portée, la mise en place progressive d'un état de résistance à l'insuline en fin de gestation chez la mère a été décrite dans de nombreuses espèces (Leturque et al 1987). Cette situation permet d'augmenter la part de glucose transféré vers les fotus au détriment des tissus maternels, qui utilisent alors davantage d'autres substrats énergétiques, tels les acides gras. L'évolution des profils postprandiaux de glucose, d'insuline et d'acides gras libres, et celle de la tolérance au glucose au cours de la gestation chez la truie, montrent qu'une diminution de la sensibilité à l'insuline se développe également dans cette espèce à partir de 85 jours de gestation, et que cet état s'accentue jusqu'au terme (figure 3 ). Cette adaptation semble cependant plus limitée que dans les autres espèces : la quantité d'insuline sécrétée reste constante et les

Tableau 2. Relations nutritionnelles materno-fœtales (Père 1995).

\begin{tabular}{|l|c|c|c|c|c|c|}
\hline & \multicolumn{3}{|c|}{ Truie } & \multicolumn{3}{c|}{ Fœtus } \\
\cline { 2 - 7 } & Artère & Veine & Extraction $^{(1)}$ & Veine & Artère & Extraction $^{(2)}$ \\
\hline Glucose $(\mathrm{mmol} / \mathrm{l})$ & 5,00 & 4,74 & $0,26^{* * *}$ & 2,30 & 2,00 & $0,31^{* * * *}$ \\
Insuline $(\mu \mathrm{U} / \mathrm{ml})$ & 16,4 & 14,9 & $1,4^{* *}$ & - & - & - \\
Lactate $(\mathrm{mmol} / \mathrm{l})$ & 0,90 & 0,88 & 0,03 & 2,49 & 2,33 & $0,16^{* * *}$ \\
Fructose $(\mathrm{mmol} / \mathrm{l})$ & - & - & - & 4,59 & 4,57 & $-0,01$ \\
NEFA $(\mu \mathrm{mol} / \mathrm{l})$ & 622 & 620 & 0 & 105 & 89 & $16^{* *}$ \\
\hline
\end{tabular}

(1) Concentration artérielle - concentration veineuse.

(2) Concentration dans la veine ombilicale - concentration dans l'artère ombilicale.
Figure 3. Evolution de la demi-vie du glucose au cours de la gestation chez des truies gravides ou chez des truies non gravides (Etienne et al 1997).

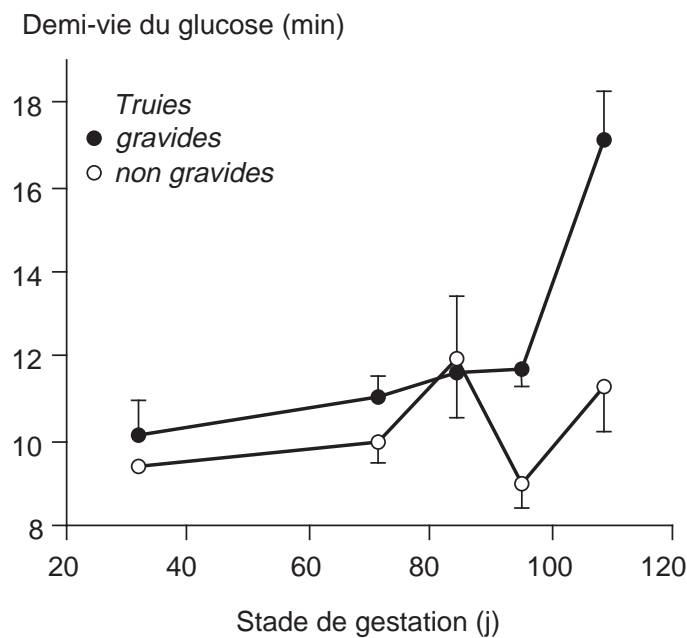

concentrations des acides gras libres n'augmentent qu'en dernière semaine de gestation. Le porc est d'ailleurs une espèce particulièrement sensible à l'insuline et des résultats préliminaires semblent montrer que la truie multipare l'est encore plus que la nullipare. Ces particularités spécifiques, jointes à la faible perméabilité placentaire aux substrats, notamment aux acides gras, contribuent à expliquer les réserves énergétiques réduites du porc nouveau-né.

\section{Références}

Duée PH. Sève B. 1978. Alimentation azotée du porc. Journées Rech. Porcine en France, 10, 167-208.

Etienne M., Père M.-C., Dourmad J.-Y., 1997. Adaptations du métabolisme glucidique chez la truie multipare. Effets de la gestation et du niveau d'alimentation. Journées Rech. Porcine en France, 29, 73-80.

Ford S.P., Reynolds L.P., Ferrell C.L., 1984. Blood flow, steroid secretion and nutrient uptake of the gravid uterus during periparturient period in sows. J. Anim. Sci., 59, 1085-1091.

Henry Y., Etienne M., 1978. Alimentation énergétique du porc. Journées Rech. Porcine en France, 10 119-166.

Leturque A., Haughel S., Ferré P., Girard J., 1987. Glucose metabolism in pregnancy. Biol. Neonate, 51 , 64-69.

Père M.-C., 1995. Maternal and fetal blood levels of glucose, lactate, fructose, and insulin in the conscious pig. J. Anim. Sci., 73, 2994-2999.

Père M.-C., 1997. Effects of meal intake on maternofotal exchanges of glucose in the pig. In : J.P. Laplace, C. Février and A. Barbeau (eds), Digestive physiology in the pig, EAAP Publication, $\mathrm{n}^{\square} 88,180$ 184. INRA, Paris. 
Père M.-C., Dourmad J.-Y., Etienne M., 1995. Mise en évidence de la capacité utérine chez la truie. Journées Rech. Porcine en France, 27, 19-24.

Père M.-C., Dourmad J.-Y., Etienne M., 1996. Variation du débit sanguin utérin au cours de la gestation chez la truie. Journées Rech. Porcine en France, 28, 371-378.
Père M.-C., Dourmad J.-Y., Etienne M., 1997. Effect of number of pig embryos in the uterus on their survival and development and on maternal metabolism. J. Anim. Sci., 75, 1337-1342.

Reynolds L.P., Ford S.P., Ferrell C.L., 1985. Blood flow and steroid and nutrient uptake of the gravid uterus and fetus of sows. J. Anim. Sci., 61, 968-974.

\section{Conséquences de l'augmentation de la prolificité des truies sur la survie et la croissance du porcelet}

La prolificité des truies et le nombre de porcelets sevrés par truie et par an constituent le premier facteur de rentabilité d'un élevage de porcs. Les cochettes d'origine hyperprolifique sont en nombre croissant dans les élevages de production et permettent actuellement un gain de $10 \%$ par rapport aux contemporaines sur la taille de portée à la naissance. Ainsi, $18,5 \%$ des portées nées en 1995/1996 avaient une taille supérieure à 15 porcelets (J. Dagorn, communication personnelle). Mais ce progrès génétique considérable ne sera pleinement valorisé que lorsque l'on parviendra à mieux maîtriser la survie postnatale et la croissance des porcelets en surnombre. Après un bref rappel des principaux facteurs associés à une mortalité postnatale élevée, le texte présente les conséquences de l'augmentation de la taille de la portée sur la survie et la croissance des porcelets ainsi que les solutions envisageables pour mieux gérer les porcelets surnuméraires, avant de conclure sur les pistes de recherche à privilégier à l'avenir.

\section{1 / Mortalité néonatale}

Malgré l'amélioration des techniques d'élevage et l'approfondissement des connaissances en matière de nutrition et de physiologie de la truie et du porcelet, la mortalité néonatale est restée particulièrement élevée dans l'espèce porcine. Entre 1975 et 1994, elle a chuté de 18,7 à $17,9 \%$ des porcelets nés et de 15 à $12,7 \%$ des nés vivants. Ces chiffres masquent cependant une très importante hétérogénéité entre les élevages, les meilleurs éleveurs réussissant à réduire ces chiffres de 3 à 4 points. Dans plus des deux tiers des cas, cette mortalité se manifeste au cours des deux premiers jours de vie et est associée aux problèmes d'écrasement et de faiblesse des porcelets : animaux légers, de faible vitalité et/ou consommant une quantité insuffisante de colostrum (J. Dagorn, communication personnelle).

Les facteurs prédisposant le nouveau-né à une mortalité précoce ne seront que briève- ment rappelés, de nombreuses synthèses ayant été publiées sur le sujet (voir par exemple Herpin et Le Dividich 1995). La survie du porcelet dépend à la fois : 1 ) de son état physiologique à la naissance (poids et maturité) ; 2) de l'homogénéité des poids intra-portée, afin d'éviter une compétition excessive entre les animaux ; 3) des conditions de mise bas, une parturition prolongée s'accompagnant d'un risque accru d'asphyxie ou d'hypoxie transitoire ; 4) des conditions d'ambiance au sein de la case de mise bas, la sensibilité du nouveau-né au froid nécessitant son maintien à une température de $33-34{ }^{\circ} \mathrm{C}$ au cours du premier jour de vie ; 5) de son aptitude à ingérer rapidement une quantité suffisante de colostrum, source d'immunoglobulines et d'énergie ; et enfin 6) des qualités maternelles et des aptitudes laitières de la truie, afin de réduire les problèmes d'écrasement et d'optimiser la production de colostrum et de lait.

Qu'en est-il dans les lignées hyperprolifiques ? A notre connaissance, aucune étude scientifique ne s'est pour le moment spécifiquement attachée à préciser les conséquences de l'hyperprolificité sur la survie et la croissance des nouveau-nés. Nous tentons ci-dessous de décrire et d'analyser ces conséquences sur la base de quelques données expérimentales sur l'effet de la taille de la portée et des observations faites actuellement dans les élevages.

\section{2 / Conséquences de l'augmentation de la taille de la portée sur la survie et la croissance du porcelet}

L'augmentation de la taille de la portée s'accompagne d'une augmentation des pertes et d'une réduction de la croissance des porcelets, particulièrement marquées pour les portées de taille supérieure à 12 (Nielsen 1981 , Legault 1985). Ceci est confirmé par une
P. HERPIN, J. LE DIVIDICH

INRA Station de Recherches Porcines, 35590 St-Gilles 